\title{
TENSOR-CLOSED OBJECTS IN THE BGG CATEGORY OF A QUANTIZED SEMISIMPLE LIE ALGEBRA
}

\author{
Zhaoting Wei \\ Received: 26 March 2020; Revised: 9 July 2020; Accepted: 15 July 2020 \\ Communicated by Burcu Üngör

\begin{abstract}
We consider the BGG category $\mathcal{O}$ of a quantized universal enveloping algebra $U_{q}(\mathfrak{g})$. We call a module $M \in \mathcal{O}$ tensor-closed if $M \otimes N \in \mathcal{O}$ for any $N \in \mathcal{O}$. In this paper we prove that $M \in \mathcal{O}$ is tensor-closed if and only if $M$ is finite dimensional. The method used in this paper applies to the unquantized case as well.
\end{abstract}

Mathematics Subject Classification (2020): 17B37, 17B10, 16T20

Keywords: BGG category, quantized universal enveloping algebra, tensor product, formal character

\section{Introduction}

BGG category $\mathcal{O}$ plays a central role in representation theory, see [3]. For a complex semisimple Lie algebra $\mathfrak{g}$ we can consider its quantized universal enveloping algebra $U_{q}(\mathfrak{g})$ and the category $\mathcal{O}$ of $U_{q}(\mathfrak{g})$ as in [1] and [7].

The large category $U_{q}(\mathfrak{g})$-Mod has a tensor product but category $\mathcal{O}$ is not closed under the tensor product. We call a module $M \in \mathcal{O}$ tensor-closed if $M \otimes N \in \mathcal{O}$ for any $N \in \mathcal{O}$. It is easy to show that finite dimensional modules are tensor-closed. Actually in [6] the authors used tensor products of finite dimensional $U_{q}(\mathfrak{g})$-modules to construct the coordinate ring of the deformed flag variety of $\mathfrak{g}$. Therefore it is interesting to ask whether we can characterize finite dimensional $U_{q}(\mathfrak{g})$-modules in a categorical way, to which we give an affirmative answer in this paper.

For (unquantized) complex semisimple Lie algebra $\mathfrak{g}$ it is a folklore theorem that any tensor-closed module in $\mathcal{O}$ must be finite dimensional, see [4] for an outline of the proof.

The main result of this paper is Theorem 4.4, which claims that $M \in \mathcal{O}$ of $U_{q}(\mathfrak{g})$ is tensor-closed if and only if $M$ is finite dimensional. This result gives a categorical characterization of finite dimensional modules in category $\mathcal{O}$. The proof is based on the idea in [4] together with a careful study of rational expressions of formal 
characters of modules in $\mathcal{O}$. We can apply the same proof to the unquantized case with little modification.

\section{A review of the $B G G$ category $\mathcal{O}$ of a quantized universal enveloping algebra}

2.1. A review of quantized universal enveloping algebras. We follow the notations in [7]. Please also see [1] for references. Let $\mathfrak{g}$ a semisimple Lie algebra over $\mathbb{C}$ of rank $N$. We fix a Cartan subalgebra $\mathfrak{h} \subset \mathfrak{g}$. Let $\boldsymbol{\Delta}$ be the set of roots and we fix $\boldsymbol{\Sigma}=\left\{\alpha_{1}, \ldots, \alpha_{N}\right\} \subset \boldsymbol{\Delta}$ the set of simple roots. We write $($,$) for the bilinear$ form on $\mathfrak{h}^{*}$ obtained by rescaling the Killing form such that the shortest root $\alpha$ of $\mathfrak{g}$ satisfies $(\alpha, \alpha)=2$. For a $\operatorname{root} \beta \in \Delta$ we set $d_{\beta}=(\beta, \beta) / 2$ and let $\beta^{\vee}=\beta / d_{\beta}$ be the corresponding coroot. In particular let $d_{i}=\left(\alpha_{i}, \alpha_{i}\right) / 2$ and hence $\alpha_{i}^{\vee}=d_{i}^{-1} \alpha_{i}$ for $i=1, \ldots, N$.

Denote by $\varpi_{1}, \ldots, \varpi_{N}$ the fundamental weights of $\mathfrak{g}$, satisfying the relations $\left(\varpi_{i}, \alpha_{j}^{\vee}\right)=\delta_{i j}$. We write

$$
\mathbf{P}=\bigoplus_{j=1}^{N} \mathbb{Z} \varpi_{j}, \quad \mathbf{Q}=\bigoplus_{j=1}^{N} \mathbb{Z} \alpha_{j}, \quad \mathbf{Q}^{\vee}=\bigoplus_{j=1}^{N} \mathbb{Z} \alpha_{j}^{\vee},
$$

for the weight, root and coroot lattices of $\mathfrak{g}$, respectively. It is well-known that $\beta^{\vee} \in \mathbf{Q}^{\vee}$ for each $\beta \in \boldsymbol{\Delta}$.

Let $\mathbf{P}^{+}$denote the set of dominant integral weights and $\mathbf{Q}^{+}$denote the set of non-negative integer combinations of the simple roots. Let $\boldsymbol{\Delta}^{+}=\mathrm{Q}^{+} \cap \boldsymbol{\Delta}$ be the set of positive roots.

As in the standard notation, let $\left(a_{i j}\right)_{1 \leq i, j \leq N}$ be the Cartan matrix for $\mathfrak{g}$ and let $W$ be the Weyl group for $\mathfrak{g}$. See $[2$, Chapter III] for details.

Definition 2.1. [7, Definition 2.13] Let $q=e^{h} \in \mathbb{R}^{\times}$be an invertible element for $h \in \mathbb{R}^{\times}$. It is clear $q$ is not a root of 1 . The algebra $U_{q}(\mathfrak{g})$ over $\mathbb{C}$ has generators $K_{\lambda}$ for $\lambda \in \mathbf{P}$, and $E_{i}, F_{i}$ for $i=1, \ldots, N$, and the defining relations for $U_{q}(\mathfrak{g})$ are

$$
\begin{gathered}
K_{0}=1, K_{\lambda} K_{\mu}=K_{\lambda+\mu}, K_{\lambda} E_{j} K_{\lambda}^{-1}=q^{\left(\lambda, \alpha_{j}\right)} E_{j}, K_{\lambda} F_{j} K_{\lambda}^{-1}=q^{-\left(\lambda, \alpha_{j}\right)} F_{j}, \\
{\left[E_{i}, F_{j}\right]=\delta_{i j} \frac{K_{i}-K_{i}^{-1}}{q_{i}-q_{i}^{-1}},}
\end{gathered}
$$

for all $\lambda, \mu \in \mathbf{P}$ and all $i, j$, together with the quantum Serre relations

$$
\begin{aligned}
& \sum_{k=0}^{1-a_{i j}}(-1)^{k}\left[\begin{array}{c}
1-a_{i j} \\
k
\end{array}\right]_{q_{i}} E_{i}^{1-a_{i j}-k} E_{j} E_{i}^{k}=0 \\
& \sum_{k=0}^{1-a_{i j}}(-1)^{k}\left[\begin{array}{c}
1-a_{i j} \\
k
\end{array}\right]_{q_{i}} F_{i}^{1-a_{i j}-k} F_{j} F_{i}^{k}=0 .
\end{aligned}
$$


In the above formulas we abbreviate $K_{i}=K_{\alpha_{i}}$ for all simple roots, and we use the notation $q_{i}=q^{d_{i}}$.

$U_{q}(\mathfrak{g})$ is a Hopf algebra with comultiplication $\hat{\Delta}: U_{q}(\mathfrak{g}) \rightarrow U_{q}(\mathfrak{g}) \otimes U_{q}(\mathfrak{g})$ given by

$$
\begin{aligned}
\hat{\Delta}\left(K_{\lambda}\right) & =K_{\lambda} \otimes K_{\lambda}, \\
\hat{\Delta}\left(E_{i}\right) & =E_{i} \otimes K_{i}+1 \otimes E_{i} \\
\hat{\Delta}\left(F_{i}\right) & =F_{i} \otimes 1+K_{i}^{-1} \otimes F_{i},
\end{aligned}
$$

counit $\hat{\epsilon}: U_{q}(\mathfrak{g}) \rightarrow \mathbb{C}$ given by $\hat{\epsilon}\left(K_{\lambda}\right)=1, \hat{\epsilon}\left(E_{j}\right)=0, \hat{\epsilon}\left(F_{j}\right)=0$, and antipode $\hat{S}$ : $U_{q}(\mathfrak{g}) \rightarrow U_{q}(\mathfrak{g})$ given by $\hat{S}\left(K_{\lambda}\right)=K_{-\lambda}, \hat{S}\left(E_{j}\right)=-E_{j} K_{j}^{-1}, \hat{S}\left(F_{j}\right)=-K_{j} F_{j}$.

Let $U_{q}\left(\mathfrak{n}_{+}\right)$be the subalgebra of $U_{q}(\mathfrak{g})$ generated by the elements $E_{1}, \ldots, E_{N}$, and $U_{q}\left(\mathfrak{b}_{+}\right)$be the subalgebra of $U_{q}(\mathfrak{g})$ generated by $E_{1}, \ldots, E_{N}$ and all $K_{\lambda}$ for $\lambda \in \mathbf{P}$. We define $U_{q}\left(\mathfrak{n}_{-}\right)$and $U_{q}\left(\mathfrak{b}_{-}\right)$in the same way. Moreover we let $U_{q}(\mathfrak{h})$ be the subalgebra generated by the elements $K_{\lambda}$ for $\lambda \in \mathbf{P}$. These algebras are Hopf subalgebras of $U_{q}(\mathfrak{g})$. By [7, Proposition 2.14] we know that there is a linear isomorphism

$$
U_{q}\left(\mathfrak{n}_{-}\right) \otimes U_{q}(\mathfrak{h}) \otimes U_{q}\left(\mathfrak{n}_{+}\right) \cong U_{q}(\mathfrak{g})
$$

2.2. A review of the BGG category $\mathcal{O}$. Recall that $1 \neq q=e^{h}$ for an $h \in \mathbb{R}^{\times}$. We shall also use the notation $\hbar=\frac{h}{2 \pi}$ hence $q=e^{2 \pi \hbar}$.

As in $\left[7\right.$, Section 2.3.1] we let $\mathfrak{h}^{*}=\operatorname{Hom}_{\mathbb{C}}(\mathfrak{h}, \mathbb{C})$ and $\mathfrak{h}_{q}^{*}=\mathfrak{h}^{*} / i \hbar^{-1} \mathbf{Q}^{\vee}$ be the parameter space for weights. Here $i=\sqrt{-1}$. It is clear that there is an embedding $\operatorname{Span}_{\mathbb{R}} \boldsymbol{\Delta} \subset \mathfrak{h}_{q}^{*}$. In particular $\mathbf{Q} \subset \mathbf{P} \subset \mathfrak{h}_{q}^{*}$.

One says that a vector $v$ in a left $U_{q}(\mathfrak{g})$-module is a weight vector of weight $\lambda \in \mathfrak{h}_{q}^{*}$ if it is a common eigenvector for the action of $U_{q}(\mathfrak{h})$ with

$$
K_{\mu} \cdot v=q^{(\lambda, \mu)} v, \quad \text { for all } \mu \in \mathbf{P} .
$$

It is well defined: if $\lambda \in i \hbar^{-1} \mathbf{Q}^{\vee}$ then for any $\mu \in \mathbf{P}$ we have $q^{(\lambda, \mu)}=e^{2 \pi \hbar(\lambda, \mu)}=1$.

Definition 2.2. ([1, Definition 3.1], [7, Definition 4.1]) A left module $M$ over $U_{q}(\mathfrak{g})$ is said to belong to the $B G G$ category $\mathcal{O}$ if

a) $M$ is finitely generated as a $U_{q}(\mathfrak{g})$-module.

b) $M$ is a weight module, that is, a direct sum of its weight spaces $M_{\lambda}$ for $\lambda \in \mathfrak{h}_{q}^{*}$.

c) The action of $U_{q}\left(\mathfrak{n}_{+}\right)$on $M$ is locally nilpotent, that is, for each $v \in M$, the subspace $U_{q}\left(\mathfrak{n}_{+}\right) \cdot v$ of $M$ is finite dimensional.

Morphisms in category $\mathcal{O}$ are all $U_{q}(\mathfrak{g})$-linear maps.

We list some basic properties of category $\mathcal{O}$. 
Proposition 2.3. (1) $\mathcal{O}$ is closed under submodules, quotient modules, and finite direct sums.

(2) All weight spaces of $M$ in $\mathcal{O}$ are finite dimensional.

(3) All finite dimensional weight modules of $U_{q}(\mathfrak{g})$ are in $\mathcal{O}$.

Definition 2.4. [7, Definition 2.31] The Verma module $M(\lambda)$ associated to $\lambda \in \mathfrak{h}_{q}^{*}$ is the induced $U_{q}(\mathfrak{g})$-module

$$
M(\lambda)=U_{q}(\mathfrak{g}) \otimes_{U_{q}\left(\mathfrak{b}_{+}\right)} \mathbb{C}_{\lambda}
$$

where $\mathbb{C}_{\lambda}$ denotes the one-dimensional $U_{q}\left(\mathfrak{b}_{+}\right)$-module $\mathbb{C}$ with the action induced from the character $\chi_{\lambda}$ determined by

$$
\chi_{\lambda}\left(K_{\mu}\right)=q^{(\lambda, \mu)} \text { for all } \mu \in \mathbf{P} \text {, and } \chi_{\lambda}\left(E_{i}\right)=0, i=1, \ldots, N .
$$

It is clear that $M(\lambda)$ belongs to category $\mathcal{O}$.

$M(\lambda)$ contains a unique maximal proper submodule $I(\lambda)$, namely the linear span of all submodules not containing the highest weight vector $v_{\lambda}=1 \otimes 1 \in M(\lambda)$. The resulting simple quotient module $M(\lambda) / I(\lambda)$ will be denoted by $V(\lambda)$. It is again a module in $\mathcal{O}$.

Remark 2.5. In [1], $M(\lambda)$ and $V(\lambda)$ are denoted by $\Delta_{q}(\lambda)$ and $L_{q}(\lambda)$ respectively.

It is clear that every highest weight module of highest weight $\lambda$ is isomorphic to a quotient of $M(\lambda)$ and every simple highest weight module of highest weight $\lambda$ is isomorphic to $V(\lambda)$.

The following result characterizes finite dimensional weight modules of $U_{q}(\mathfrak{g})$

Proposition 2.6. $\left[7\right.$, Corollary 2.100] We write $\mathbf{X}_{q}$ for the set of weights $\omega \in \mathfrak{h}_{q}^{*}$ satisfying $q^{(\omega, \alpha)}= \pm 1$ for all $\alpha \in \mathbf{Q}$. We define

$$
\mathbf{P}_{q}^{+}=\mathbf{P}^{+}+\mathbf{X}_{q} \subset \mathfrak{h}_{q}^{*} .
$$

Then every finite dimensional weight module over $U_{q}(\mathfrak{g})$ decomposes into a direct sum of irreducible highest weight modules $V(\lambda)$ for weights $\lambda \in \mathbf{P}_{q}^{+}$.

Simple modules $V(\lambda)$ are the building blocks of modules in $\mathcal{O}$.

Proposition 2.7. [7, Theorem 4.3] Every module $M \in \mathcal{O}$ is both Artinian and Noetherian. Hence every module $M \in \mathcal{O}$ has a Jordan-Hölder decomposition series $0=M_{0} \subset M_{1} \subset \cdots \subset M_{n}=M$ such that all subquotients $M_{j+1} / M_{j}$ are simple highest weight modules. Moreover, the number of subquotients isomorphic to $V(\lambda)$ for $\lambda \in \mathfrak{h}_{q}^{*}$ is independent of the decomposition series and will be denoted by $[M: V(\lambda)]$. 
To further study $[M(\mu): V(\lambda)]$ for a Verma module $M(\mu)$ we need the following concept.

Definition 2.8. ([5, Section 8.3.2], [7, Definition 2.125]) We define

$$
\mathbf{Y}_{q}=\left\{\zeta \in \mathfrak{h}_{q}^{*} \mid 2 \zeta=0\right\} \cong \frac{1}{2} i \hbar^{-1} \mathbf{Q}^{\vee} / i \hbar^{-1} \mathbf{Q}^{\vee}
$$

It is clear that $W$ acts on $\mathbf{Y}_{q}$. The extended Weyl group $\hat{W}$ is defined as the semidirect product

$$
\hat{W}=\mathbf{Y}_{q} \rtimes W
$$

with respect to the action of $W$ on $\mathbf{Y}_{q} \cdot \hat{W}$ is a finite group.

Explicitly, the product in $\hat{W}$ is $(i \zeta, v)(i \eta, w)=(i \zeta+i v \eta, v w)$. We define two actions of $\hat{W}$ on $\mathfrak{h}_{q}^{*}$ by $(i \zeta, w) \lambda=w \lambda+i \zeta$ and

$$
(i \zeta, w) \cdot \lambda=w \cdot \lambda+i \zeta=w(\lambda+\rho)-\rho+i \zeta
$$

for $\lambda \in \mathfrak{h}_{q}^{*}$, where $\rho$ is the half sum of all positive roots. The latter is called the shifted action of $\hat{W}$ on $\mathfrak{h}_{q}^{*}$.

Remark 2.9. See Theorem 2.128 of [7] for the relation between the $\frac{1}{2} i \hbar^{-1} \mathbf{Q}^{\vee}$-translation and the Harish-Chandra map, which plays an important role in the representation theory of $U_{q}(\mathfrak{g})$.

Definition 2.10. We say that $\mu, \lambda \in \mathfrak{h}_{q}^{*}$ are $\hat{W}$-linked if $\hat{w} \cdot \lambda=\mu$ for some $\hat{w} \in \hat{W}$.

Definition 2.11. We define a partial order $\geq$ on $\mathfrak{h}_{q}^{*}$ by saying that $\lambda \geq \mu$ if $\lambda-\mu \in \mathbf{Q}^{+}$. Here we are identifying $\mathbf{Q}^{+}$with its image in $\mathfrak{h}_{q}^{*}$.

Lemma 2.12. [7, Section 4.1.1] For any $\mu \in \mathfrak{h}_{q}^{*}$ we have $[M(\mu): V(\mu)]=1$, and moreover $[M(\mu): V(\lambda)]=0$ unless $\lambda \leq \mu$ and $\lambda$ is $\hat{W}$-linked to $\mu$. Since $\hat{W}$ is a finite group, for each $\mu \in \mathfrak{h}_{q}^{*}$ there exists only finitely many $\lambda \in \mathfrak{h}_{q}^{*}$ such that $[M(\mu): V(\lambda)] \neq 0$.

\section{Formal characters of modules in category $\mathcal{O}$}

\subsection{Basic properties of formal characters.}

Definition 3.1. We define the formal character of $M$ in $\mathcal{O}$ as the formal sum

$$
\operatorname{ch}(M)=\sum_{\lambda \in \mathfrak{h}_{q}^{*}} \operatorname{dim}\left(M_{\lambda}\right) e^{\lambda}
$$

By Proposition 2.3 any module $M$ in category $\mathcal{O}$ satisfies $\operatorname{dim} M_{\lambda}<\infty$ for all $\lambda \in \mathfrak{h}_{q}^{*}$. So $\operatorname{ch}(M)$ is well-defined. We also have the following more general definition: 
Definition 3.2. Let $\mathcal{X}$ be the ring of formal sums of the form $\sum_{\lambda \in \mathfrak{h}_{q}^{*}} f(\lambda) e^{\lambda}$ where $f: \mathfrak{h}_{q}^{*} \rightarrow \mathbb{Z}$ is any integer valued function whose support lies in a finite union of sets of the form $\nu-\mathbf{Q}^{+}$with $\nu \in \mathfrak{h}_{q}^{*}$. The product in $\mathcal{X}$ is the convolution product given by

It is clear that the right hand side is still in $\mathcal{X}$.

$$
\left(\sum_{\lambda \in \mathfrak{h}_{q}^{*}} f(\lambda) e^{\lambda}\right)\left(\sum_{\mu \in \mathfrak{h}_{q}^{*}} g(\mu) e^{\mu}\right)=\sum_{\lambda, \mu \in \mathfrak{h}_{q}^{*}} f(\lambda) g(\mu) e^{\lambda+\mu} .
$$

Definition 3.3. We introduce an element $p \in \mathcal{X}$ as

$$
p=\prod_{\beta \in \Delta^{+}}\left(\sum_{m=0}^{\infty} e^{-m \beta}\right) .
$$

Lemma 3.4. [7, Proposition 2.68] For each $\mu \in \mathfrak{h}_{q}^{*}$, the formal character of the Verma module $M(\mu)$ is the convolution product of $e^{\mu}$ and $p$ :

$$
\operatorname{ch}(M(\mu))=e^{\mu} p .
$$

By Lemma 2.12 we have

$$
\operatorname{ch}(M(\mu))=\sum_{\lambda \in \mathfrak{h}_{q}^{*}}[M(\mu): V(\lambda)] \operatorname{ch}(V(\lambda)) .
$$

where $[M(\mu): V(\mu)]=1$ and $[M(\mu): V(\lambda)]=0$ unless $\lambda \leq \mu$ and $\lambda$ is $\hat{W}$-linked to $\mu$.

We can obtain the following well-known result on $\operatorname{ch}(V(\mu))$ by inverting the matrix $[M(\mu): V(\lambda)]$ :

Lemma 3.5. For each $\mu \in \mathfrak{h}_{q}^{*}$, the formal character of the simple highest weight module $V(\mu)$ can be expressed as

$$
\operatorname{ch}(V(\mu))=\sum_{\lambda \in \mathfrak{h}_{q}^{*}} m_{\lambda, \mu} \operatorname{ch}(M(\lambda))=\sum_{\lambda \in \mathfrak{h}_{q}^{*}} m_{\mu, \lambda} e^{\lambda} p
$$

where $m_{\mu, \lambda}$ are integers such that $m_{\mu, \mu}=1$ and $m_{\mu, \lambda}=0$ unless $\lambda \leq \mu$ and $\lambda$ is $\hat{W}$-linked to $\mu$.

Remark 3.6. If $\mu \in \mathbf{P}^{+}$the set of dominant integral weights, then [7, Proposition 4.4] gives a more precise formula than (15).

Corollary 3.7. For each $M \in \mathcal{O}$, there exists a finite set $\left\{\mu_{1}, \ldots, \mu_{m}\right\} \subset \mathfrak{h}_{q}^{*}$ such that

$$
\operatorname{ch}(M)=\sum_{i=1}^{m} \sum_{\lambda \in \mathfrak{h}_{q}^{*}}\left[M: V\left(\mu_{i}\right)\right] m_{\mu_{i}, \lambda} e^{\lambda} p .
$$

where $m_{\mu_{i}, \lambda}$ are integers such that $m_{\mu_{i}, \mu_{i}}=1$ and $m_{\mu_{i}, \lambda}=0$ unless $\lambda \leq \mu_{i}$ and $\lambda$ is $\hat{W}$-linked to $\mu_{i}$ 
Remark 3.8. Since $\hat{W}$ is a finite group, the sums on the right hand side of (15) and (16) are both finite.

3.2. Reduced rational expressions of formal characters of modules in $\mathcal{O}$. Notice that we can write the formal character $p=\prod_{\beta \in \Delta^{+}}\left(\sum_{m=0}^{\infty} e^{-m \beta}\right)$ as

$$
p=\frac{1}{\prod_{\beta \in \Delta^{+}}\left(1-e^{-\beta}\right)}
$$

so by Corollary 3.7 for each $M \in \mathcal{O}$, we can write its formal character as

$$
\operatorname{ch}(M)=\frac{\sum_{i=1}^{m} \sum_{\lambda \in \mathfrak{h}_{q}^{*}}\left[M: V\left(\mu_{i}\right)\right] m_{\mu_{i}, \lambda} e^{\lambda}}{\prod_{\beta \in \Delta^{+}}\left(1-e^{-\beta}\right)}
$$

We want to simplify $\operatorname{ch}(M)$ to obtain a reduced fraction, which needs some work because the ring $\mathcal{X}$ is not a UFD.

Let $\mathcal{S}$ be the ring of $\mathbb{Z}$-coefficient polynomials generated by $e^{-\alpha_{i}}, i=1, \ldots, N$, where $\left\{\alpha_{1}, \ldots, \alpha_{N}\right\}$ is the set of simple roots. It is clear that $\prod_{\beta \in \Delta^{+}}\left(1-e^{-\beta}\right) \in \mathcal{S}$ but

$$
\sum_{i=1}^{m} \sum_{\lambda \in \mathfrak{h}_{q}^{*}}\left[M: V\left(\mu_{i}\right)\right] m_{\mu_{i}, \lambda} e^{\lambda}
$$

is not necessarily contained in $\mathcal{S}$.

We have the following definition.

Definition 3.9. Let $\mathcal{X}$ be as in Definition 3.2. We say that $a \in \mathcal{X}$ can be written in reduced rational form if there exists a subset $T_{a} \subset \boldsymbol{\Delta}^{+}$and a finite collection $\left\{\mu_{1}, \ldots, \mu_{m}\right\} \subset \mathfrak{h}_{q}^{*}$ such that

$$
a=\frac{\sum_{i=1}^{m} e^{\mu_{i}} f_{i}}{\prod_{\beta \in T_{a}}\left(1-e^{-\beta}\right)^{n_{\beta}}}
$$

where

(1) $\mu_{i}-\mu_{j}$ is not in the root lattice $\mathbf{Q}$ for each $i \neq j$;

(2) $f_{i}$ is a polynomial in $\mathcal{S}$ with nonzero constant term for each $i$;

(3) $n_{\beta}$ is a positive integer for each $\beta \in T_{a}$;

(4) The numerator and denominator of (19) are coprime. More precisely, for each $\beta \in T_{a}$, there exists an $f_{i}$ in the numerator such that $1-e^{-\beta}$ is not a factor of $f_{i}$.

We call the set $T_{a}$ the denominator roots of $a$.

Lemma 3.10. For any $a \in \mathcal{X}$, the reduced rational form of a is unique if exists.

Proof. It is clear from the definition and the fact that the polynomial ring $\mathcal{S}$ is a UFD. 
Not all elements in $\mathcal{X}$ can be written in reduced rational form. Nevertheless for formal characters of modules in $\mathcal{O}$ we have the following result.

Lemma 3.11. Let $M \in \mathcal{O}$ be a nonzero module. Then the set $T_{c h(M)}$ of denominator roots exists and $\mathrm{ch}(M)$ can be written uniquely in reduced rational form. Moreover we have

$$
\operatorname{ch}(M)=\frac{\sum_{i=1}^{m} e^{\mu_{i}} f_{i}}{\prod_{\beta \in T_{\operatorname{ch}(M)}}\left(1-e^{-\beta}\right)}
$$

and Property 1, 2, 3, 4 in Definition 3.9 are satisfied with all $n_{\beta}=1$. In the sequel we will denote $T_{c h(M)}$ by $T_{M}$ and we will call $T_{M}$ the set of denominator roots of $M$.

Proof. It is a direct consequence of Corollary 3.7, Lemma 3.10, and (18).

Example 3.12. By Lemma 3.11, for any $\alpha \in \boldsymbol{\Delta}^{+}$the formal power series

$$
\frac{1}{\left(1-e^{-\alpha}\right)^{2}}
$$

cannot be the formal character of any module in $\mathcal{O}$ although $\frac{1}{\left(1-e^{-\alpha}\right)^{2}} \in \mathcal{X}$. Intuitively this is because the multiplicity of $e^{-n \alpha}$ grows too fast as $n$ grows and here we have a precise criterion of this fact.

Corollary 3.13. A nonzero module $M \in \mathcal{O}$ is finite dimensional if and only if its reduced rational form has denominator $=1$, i.e. $T_{M}=\varnothing$.

Proof. It is implied by the uniqueness of reduced rational form.

Example 3.14. For a Verma module $M(\mu)$, the reduced rational form of its formal character is

hence $T_{M(\mu)}=\boldsymbol{\Delta}^{+}$.

$$
\operatorname{ch}(M(\mu))=\frac{e^{\mu}}{\prod_{\beta \in \Delta^{+}}\left(1-e^{-\beta}\right)}
$$

Example 3.15. For a simple highest weight module $V(\mu)$ we have the reduced rational form

$$
\operatorname{ch}(V(\mu))=\frac{e^{\mu} f_{V(\mu)}}{\prod_{\beta \in T_{V(\mu)}}\left(1-e^{-\beta}\right)}
$$

for some $f_{V(\mu)} \in \mathcal{S}$. In particular if $\mu \in \mathbf{P}_{q}^{+}$then $T_{V(\mu)}=\varnothing$ and $\operatorname{ch}(V(\mu))$ is given explicitly by the Weyl character formula. Actually [7, Proposition 4.4] only covers the $\mu \in \mathbf{P}^{+}$case but the general case can be easily obtained by [7, Lemma 2.41$]$.

Remark 3.16. For any $M \in \mathcal{O}$ it is clear that the denominator roots $T_{M} \subset \cup T_{V(\mu)}$ where the union is for all $V(\mu)$ such that $[M: V(\mu)] \neq 0$. The author does not know whether we have $T_{M}=\bigcup T_{V(\mu)}$ for all $V(\mu)$ such that $[M: V(\mu)] \neq 0$. Nevertheless we do not need this result in this paper. 


\section{Tensor closed objects in category $\mathcal{O}$}

The category $U_{q}(\mathfrak{g})$-Mod has a tensor product since $U_{q}(\mathfrak{g})$ is a Hopf algebra. Moreover we have the following lemma.

Lemma 4.1. $U_{q}(\mathfrak{g})$-Mod is a braided category. In particular for any left $U_{q}(\mathfrak{g})$ modules $V$ and $W$ we have a $U_{q}(\mathfrak{g})$-module isomorphism $V \otimes W \cong W \otimes V$.

Proof. It is clear since $U_{q}(\mathfrak{g})$ is quasitriangular in the sense of [7, Theorem 2.108].

However category $\mathcal{O}$ is not closed under tensor product.

Definition 4.2. We call a module $M \in \mathcal{O}$ tensor-closed if for any $N \in \mathcal{O}$, the tensor product $M \otimes N \cong N \otimes M$ is still in $\mathcal{O}$.

The following result is well-known.

Lemma 4.3. Any finite dimensional module $V \in \mathcal{O}$ is tensor-closed.

Proof. The proof is the same as that of [3, Theorem $1.1(\mathrm{~d})]$.

In this section we prove the following result.

Theorem 4.4. A module $V \in \mathcal{O}$ is tensor-closed if and only if it is finite dimensional.

To give the proof more rigorously we introduce the following auxiliary category.

Definition 4.5. A left module $M$ over $U_{q}(\mathfrak{g})$ is said to belong to the category $\widetilde{\mathcal{O}}$ if

a) $M$ is a weight module and all weight spaces of $M$ are finite dimensional.

b) There exist finitely many weights $\nu_{1}, \ldots, \nu_{l} \in \mathfrak{h}_{q}^{*}$ such that

$$
\operatorname{supp} M \subset \bigcup_{i=1}^{l}\left(\nu_{i}-\mathbf{Q}^{+}\right),
$$

where $\operatorname{supp} M=\left\{\lambda \in \mathfrak{h}_{q}^{*} \mid M_{\lambda} \neq 0\right\}$.

Morphisms in category $\widetilde{\mathcal{O}}$ are all $U_{q}(\mathfrak{g})$-linear maps.

It is clear that $\mathcal{O}$ is a full subcategory of $\widetilde{\mathcal{O}}$. $\widetilde{\mathcal{O}}$ is closed under tensor product and modules in $\widetilde{\mathcal{O}}$ have formal characters in the ring $\mathcal{X}$ in Definition 3.2. Moreover for $M, N \in \widetilde{\mathcal{O}}$ we have

$$
\operatorname{ch}(M \otimes N)=\operatorname{ch}(M) \operatorname{ch}(N)
$$


Lemma 4.6. For two simple highest weight modules $V(\mu)$ and $V(\lambda)$, if $V(\mu) \otimes$ $V(\lambda) \in \mathcal{O}$, then

$$
T_{V(\mu)} \cap T_{V(\lambda)}=\varnothing .
$$

In particular for any infinite dimensional simple highest weight module $V(\mu)$ we have $V(\mu) \otimes V(\mu) \notin \mathcal{O}$.

Proof. For each simple highest weight module $V(\mu)$ we have the reduced rational form

$$
\operatorname{ch}(V(\mu))=\frac{e^{\mu} f_{V(\mu)}}{\prod_{\beta \in T_{V(\mu)}}\left(1-e^{-\beta}\right)}
$$

where $f_{V(\mu)}$ is in the polynomial ring $\mathcal{S}$ such that $1-e^{-\beta}$ is not a factor of $f_{V(\mu)}$ for each $\beta \in T_{V(\mu)}$. Therefore

$$
\operatorname{ch}(V(\mu) \otimes V(\lambda))=\operatorname{ch}(V(\mu)) \operatorname{ch}(V(\lambda))=\frac{e^{\mu+\lambda} f_{V(\mu)} f_{V(\lambda)}}{\prod_{\beta \in T_{V(\mu)}}\left(1-e^{-\beta}\right) \prod_{\gamma \in T_{V(\lambda)}}\left(1-e^{-\gamma}\right)} .
$$

Assume $T_{V(\mu)} \cap T_{V(\lambda)} \neq \varnothing$ and let $\beta \in T_{V(\mu)} \cap T_{V(\lambda)}$, then $\left(1-e^{-\beta}\right)^{2}$ appears in the denominator and $1-e^{-\beta}$ is not a factor of $f_{V(\mu)}$ nor $f_{V(\lambda)}$. Therefore $\left(1-e^{-\beta}\right)^{2}$ appears in the denominator of the reduced rational form of $\operatorname{ch}(V(\mu) \otimes V(\lambda))$. On the other hand by Lemma 3.11, if $V(\mu) \otimes V(\lambda)$ is in $\mathcal{O}$ then the reduced rational form of $\operatorname{ch}(V(\mu) \otimes V(\lambda))$ cannot have squares in the denominator. Hence $V(\mu) \otimes V(\lambda) \in \mathcal{O}$ implies $T_{V(\mu)} \cap T_{V(\lambda)}=\varnothing$.

For infinite dimensional $V(\mu)$, we know $T_{V(\mu)} \neq \varnothing$ by Corollary 3.13, so $V(\mu) \otimes$ $V(\mu) \notin \mathcal{O}$.

Lemma 4.7. For any infinite dimensional simple highest weight module $V(\mu)$ and any Verma module $M(\lambda)$ we have $V(\mu) \otimes M(\lambda) \cong M(\lambda) \otimes V(\mu) \notin \mathcal{O}$.

Proof. Similar to the proof of Lemma 4.6, we can show that $\operatorname{ch}(V(\mu) \otimes M(\lambda))$ has squares in the denominator of its reduced rational form.

Remark 4.8. In general the product of two reduced rational forms needs not to be a reduced rational form since $\mathcal{X}$ is not a UFD. For example for any $\alpha \in \Delta^{+}$let

$$
a=\frac{1+e^{-\alpha / 2}}{1-e^{-\alpha}}, b=\frac{1-e^{-\alpha / 2}}{1-e^{-\alpha}} .
$$

It is clear that both $a$ and $b$ are reduced rational forms but

$$
a b=\frac{1-e^{-\alpha}}{\left(1-e^{-\alpha}\right)^{2}}
$$

is not reduced. The author does not know if we restrict to formal characters of modules in $\mathcal{O}$, whether or not the product of reduced rational forms must be a reduced rational form. Nevertheless we do not need this result in this paper. 
Proof of Theorem 4.4. Let $M \in \mathcal{O}$ be infinite dimensional and we want to show that $M$ is not tensor-closed. Actually by Proposition 2.7 there exists an infinite dimensional $V(\mu)$ in the Jordan-Hölder series of $M$. By Lemma 4.6, $V(\mu) \otimes V(\mu) \notin$ $\mathcal{O}$. Since $\mathcal{O}$ is closed under subquotients, $M \otimes V(\mu) \notin \mathcal{O}$ too. So $M$ is not tensorclosed.

Remark 4.9. There exist two infinite dimensional modules with tensor product still in $\mathcal{O}$. Victor Ostrik gave the following example: Let $\mathfrak{g}=\operatorname{sl}(2) \oplus \operatorname{sl}(2)$. Let $V$ be a Verma module for $U_{q}(s l(2))$ (with arbitrary highest weight). Using two projections $\mathfrak{g} \rightarrow \operatorname{sl}(2)$ we can consider $V$ as a $U_{q}(\mathfrak{g})$-module in two different ways. Let us call the resulting $U_{q}(\mathfrak{g})$-modules $V_{1}$ and $V_{2}$. Then both $V_{1}$ and $V_{2}$ are in the category $\mathcal{O}$ for $U_{q}(\mathfrak{g})$, and $V_{1} \otimes V_{2}$ is a Verma module of $U_{q}(\mathfrak{g})$.

It is an interesting question if $\mathfrak{g}$ is simple and $M, N \in \mathcal{O}$ are both infinite dimensional, is it always true that $M \otimes N \notin \mathcal{O}$. See [8] for a discussion in the cases that $\mathfrak{g}$ is simple of type ADE.

Remark 4.10. All arguments and proofs in this paper work for the unquantized case as well. In particular we can proof Theorem 4.4 for the unquantized case using the method in this paper.

Acknowledgement. James Humphreys told the author to use formal characters to show certain modules are not in $\mathcal{O}$. Peter McNamara told the author to consider the rational form of formal characters. Victor Ostrik provided the counter-example in Remark 4.9. The author wants to thank them all. The author also wants to thank Weibo Fu for kindly answering questions related to this paper.

\section{References}

[1] H. H. Andersen and V. Mazorchuk, Category $\mathscr{O}$ for quantum groups, J. Eur. Math. Soc., 17(2) (2015), 405-431.

[2] J. E. Humphreys, Introduction to Lie Algebras and Representation Theory, Second printing, revised, Graduate Texts in Mathematics, 9, Springer-Verlag, New York-Berlin, 1978.

[3] J. E. Humphreys, Representations of Semisimple Lie Algebras in the BGG Category $\mathscr{O}$, Graduate Studies in Mathematics, 94, American Mathematical Society, Providence, RI, 2008.

[4] J. E. Humphreys, Tensor-closed objects of the BGG category $\mathscr{O}$, (2015), Preprint available on the author's website: http://people.math.umass.edu / jeh/pub/ tensor.pdf. 
[5] A. Joseph, Quantum Groups and Their Primitive Ideals, Ergebnisse der Mathematik und ihrer Grenzgebiete, (3) 29, Springer-Verlag, Berlin, 1995.

[6] V. A. Lunts and A. L. Rosenberg, Localization for quantum groups, Selecta Math. (N.S.), 5(1) (1999), 123-159.

[7] C. Voigt and R. Yuncken, Complex semisimple quantum groups and representation theory, (2017), arXiv:1705.05661.

[8] Z. Wei, Tensor products of infinite dimensional modules in the $B G G$ category of a quantized simple Lie algebra of type ADE, (2019), Preprint available on the author's website: https://drive.google.com/file/d/ 1ZogGH4Rfenq7AXTvxOdsoqGbSHCbfrjr/view.

\section{Zhaoting Wei}

Department of Mathematics

Texas A\&M University-Commerce

TX 75429, USA

e-mail: zhaoting.wei@tamuc.edu 